\title{
Emerging roles of cancer-testis antigenes, semenogelin 1 and 2, in neoplastic cells
}

\author{
Oleg Shuvalov', Alyona Kizenko (1)', Alexey Petukhov ${ }^{1,2}$, Olga Fedorova', Alexandra Daks (1) ${ }^{1}$ and Nikolai Barlev ${ }^{1,3}$
}

\begin{abstract}
Cancer-testicular Antigens (CTAs) belong to a group of proteins that under normal conditions are strictly expressed in a male's reproductive tissues. However, upon malignisation, they are frequently re-expressed in neoplastic tissues of various origin. A number of studies have shown that different CTAs affect growth, migration and invasion of tumor cells and favor cancer development and metastasis. Two members of the CTA group, Semenogelin 1 and 2 (SEMG1 and SEMG2, or SEMGs) represent the major component of human seminal fluid. They regulate the motility and capacitation of sperm. They are often re-expressed in different malignancies including breast cancer. However, there is almost no information about the functional properties of SEMGs in cancer cells. In this review, we highlight the role of SEMGs in the reproductive system and also summarize the data on their expression and functions in malignant cells of various origins.
\end{abstract}

\section{Facts}

- Cancer-testis Antigens (CTAs) are frequently expressed in different neoplasms, induce EMT and are associated with unfavorable prognosis for patient's survival

- SEMG1 and SEMG2 are two non-X linked CTAs that normally regulate motility and maturation of sperm but become re-expressed in different cancerous tissues

- SEMG1 and SEMG2 possess biological activity in tumors, can both increase energy metabolism and display anti-proliferative properties

\section{Open Questions}

- Do SEMG1 and SEMG2 undergo processing and do their functions in cancer cells depend on proteases?

- What are molecular functions of SEMG1 and SEMG2 in tumor cells with different background?

Correspondence: Nikolai Barlev (nick.a.barlev@gmail.com)

${ }^{1}$ Institute of cytology RAS, St-Petersburg, Russia

${ }^{2}$ Almazov Federal North-West Medical Research Centre, St-Petersburg, Russia

Full list of author information is available at the end of the article
- Do SEMG1 and SEMG2 elicit immune response in cancer patients?

- Is it possible to use them as tumor's biomarkers?

\section{Introduction}

Cancer-testicular antigens (CTAs) have long been considered as potential prognostic tumor markers ${ }^{1}$ and as targets for the immunotherapy of malignant tumors ${ }^{2}$. CTAs are primarily expressed in germ cells of the male's body. Moreover, they are often expressed in malignant neoplasms of various origins, and therefore they are referred to as tumor antigens. Thus, many CTAs are immunogenic markers of tumor cells, which makes them valuable targets for antitumor vaccines and therapy using reprogrammed T-cells (chimeric antigen receptors).

Semenogelin 1 and 2 (SEMG1 and SEMG2, respectively) are two autosomal CTAs. They are the major proteins of human semen and are mainly expressed by the glandular epithelium of seminal vesicles ${ }^{4}$. Several of their functions are well described: the regulation of sperm motility and capacitation, as well as an antibacterial protection of the former.

Surprisingly, although the SEMGs genes share a high degree of homology (78\%) between them, almost all the 
information available in literature about their biological role in reproduction concerns only SEMG1. Interestingly, it was shown that in addition to the tissues of reproductive organs, SEMGs can also be detected at the mRNA level in a number of other tissues-the retina, epithelium of the gastrointestinal tract, skeletal muscle, central nervous system tissues ${ }^{5}$, etc. In line with their belonging to a group of CTAs, SEMGs were found expressed in various human malignancies. So far, there is no data available on the functional role of SEMGs in neoplastic cells, except in prostate cancer.

Below we summarize the information on known functions of SEMGs in reproductive tissues, as well as data on their expression in other normal tissues and malignant neoplasms of various origins. Based on generalizations, we analyze the possible functions of SEMGs in neoplastic cells.

\section{Cancer-testicular antigens}

Tumor cells of various origins exhibit on their surface, and excrete into the bloodstream, various antigens that can be recognized by immune system, followed by the eradication of such cells. In this way, the specific antitumor immune response can be generated.

This underlies the development of antitumor vaccines aimed at the activation of specific antitumor immunity in patients. Moreover, the immune response to tumor antigens varies and is often not effective due to the presence of a number of protective mechanisms in the tumors, allowing them to escape from the immune response ${ }^{6}$.

Tumor antigens can be divided into two groups: tumorspecific and tumor-associated antigens. Tumor-specific antigens are unique antigens that are characteristic only of neoplastic cells ${ }^{7}$. Such antigens, for example, include the well-known chimeric protein $\mathrm{BCR}-\mathrm{ABL}$, which is a specific marker of acute myeloid leukemia cells.

Tumor-associated antigens are the most widely represented tumor antigens. They include proteins, which are often present on the surface of tumor cells but are also expressed by normal cells of some tissues. One of the tumor-associated antigen subgroups is cancer-testicular antigens (CTAs), which include proteins synthesized primarily in the tissues of the testis (spermatogonia, spermatids, spermatozoa), as well as in the trophoblast and placenta ${ }^{8}$. In addition, the expression of these proteins is reported in neoplastic tissues of various origins ${ }^{9}$. Their presence is often associated with low-grade tumors and an unfavorable prognosis for the patient's survival $^{10,11}$.

The main features according to which antigens are assigned to the CTAs group are the predominant expression of corresponding genes in the germ cells of the testis, their frequent expression in neoplastic cells, and the frequent localization at the $\mathrm{X}$ chromosome ${ }^{12}$. Thus, proteins can be classified as CTAs when their expression in normal is observed in germ cells but also can also be reexpressed in tumors. However, not all of these proteins are capable of eliciting immune response; nonetheless they are united by the term 'CTAs'.

Currently, the CTAs database ${ }^{13}$ (http://www.cta.lncc.br/) contains information about 204 genes from more than 100 families; the level of mRNA, protein expression and their immunogenicity. CTAs can be divided into two subgroups: ones localized at the $\mathrm{X}$ chromosome (X-linked CTAs or X-CTAs) and distributed throughout the rest of the genome (non-X-CTAs). X-CTAs coding genes are characterized by multiple copies; together they occupy about $10 \%$ of the X chromosome. X-CTAs make up about half of all CTAs and, as a rule, form clusters at the chromosome $^{14}$. They often form multi-gene families. On the contrary, non-X-CTAs coding genes are distributed throughout the genome and are most often represented by single-copy genes. X-CTAs are most often expressed in spermatogonia, while non-X-CTAs are predominantly expressed in the later stages of germ cell differentiation in spermatocytes ${ }^{8}$.

CTAs are known to have different biological properties in neoplastic cells (Fig. 1). It was shown that CTAs can provide sustained tumor growth, tissue invasion and metastasis, genomic instability, evading of apoptosis, inducing angiogenesis and so on (for comprehensive review see please ${ }^{2,15,16}$.

\section{Cancer-testicular antigens affect EMT}

The epithelial-to-mesenchymal transition (EMT) is the process of acquiring mesenchymal phenotype by epithelial cells. In normal, this process occurs during embryonic development, tissue regeneration, organ fibrosis, and wound healing ${ }^{17}$. However, in cancer, EMT is associated with metastasis formation. By undergoing EMT, cancer cells disrupt cell-cell adhesion, polarity and acquire migratory and invasive properties ${ }^{18}$. These EMTassociated events allow cancer cells to invade the bloodstream, followed by dissemination throughout the body ${ }^{19}$.

However, EMT is also reversible by the mesenchymal-toepithelial transition (MET). It is deemed to affect circulating cancer cells when they reach a desirable metastatic niche to develop secondary tumors. EMT is not only associated with the development of metastasis but also contributes to the increased resistance of neoplastic cells against multiple chemotherapeutics and radiotherapy ${ }^{20,21}$.

Since metastasis is the main reason for cancer-mediated cell death, attention of many scientists is focused on the study of mechanisms regulating EMT. Indeed, various studies have shown that CTAs both trigger EMT and contribute to the genesis of cancer stem-like cells, escalating tumorigenesis, invasion, and metastasis ${ }^{22}$. Some of these studies listed in Table 1 show that well-known 


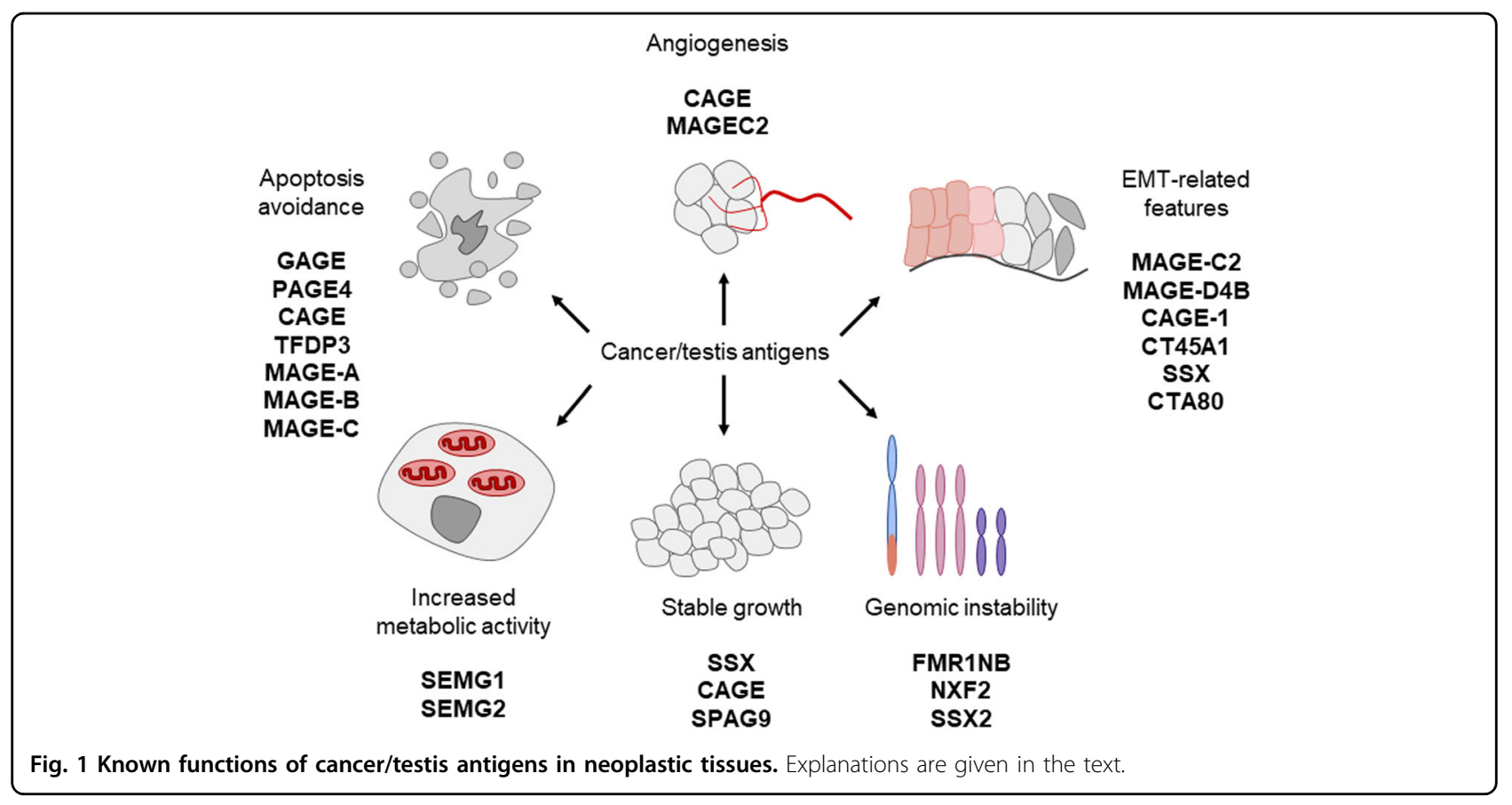

Table 1 CTAs promote EMT in neoplasms of different origin.

\begin{tabular}{|c|c|c|c|}
\hline Gene & Type of cancer & Effect on EMT and EMT-related processes? & Reference \\
\hline SPANXB1 & $\begin{array}{l}\text { Triple Negative Breast } \\
\text { Cancer (TNBC) }\end{array}$ & $\begin{array}{l}\text { Promotes migration, invasion and reactive oxygen species production of } \\
\text { TNBC cells }\end{array}$ & 26 \\
\hline $\begin{array}{l}\text { SPANX-A/C/D } \\
\text { and CTAG2 }\end{array}$ & Breast cancer & $\begin{array}{l}\text { CTAG2 is necessary for directional migration SPANX-A/C/D is required for } \\
\text { formation of actin-rich cellular protrusions that reorganize the } \\
\text { extracellular matrix }\end{array}$ & 27 \\
\hline TSP50 & Gastric cancer & $\begin{array}{l}\text { Promotes the proliferation, migration and invasion of gastric cancer cells } \\
\text { involving NF-KB dependent EMT activation }\end{array}$ & 28 \\
\hline CT45A1 & Breast cancer & $\begin{array}{l}\text { Constitutively activates ERK and CREB signaling pathways, promotes EMT, and } \\
\text { increases cell stemness, tumorigenesis, invasion, and metastasis }\end{array}$ & 29 \\
\hline MAGEC2 & Hepatocellular carcinoma & Promotes EMT & 30 \\
\hline MAGEC2 & Breast cancer & $\begin{array}{l}\text { Promotes EMT by binding to a transcriptional repressor, KAP1, which plays a } \\
\text { critical role in promoting cadherin switching and acquiring mesenchymal cell } \\
\text { molecular patterns }\end{array}$ & 23 \\
\hline SPAG9 & Ovarian cancer & $\begin{array}{l}\text { Increased the expression of mesenchymal markers and decreased expression of } \\
\text { epithelial markers }\end{array}$ & 31 \\
\hline SPAG9 & Bladder cancer & Promotes migration, decreases E-cadherin and increases Vimentin & 32 \\
\hline PIWIL1 (HIWI) & Hepatocellular carcinoma & Promotes cell migration and invasion & 33 \\
\hline PIWIL2 (HILI) & Colon cancer & Activates MMP9 and enhance migration and invasion & 34 \\
\hline SSX & Melanoma & Activates MAPK/Erk and Wnt signaling, promotes EMT & 35 \\
\hline
\end{tabular}

CTAs induce EMT, enhance migration and evasion of cancer cells in different models of breast, gastric, colon, hepatocellular, ovarian, cervical cancer, melanoma, and multiple myeloma.
Although a number of studies point to the CTAsinduced augmentation of mesenchymal markers (vimentin, N-cadherin, Snail, Slug, etc.), simultaneously with a decrease of epithelial marker E-cadherin, there is only one 


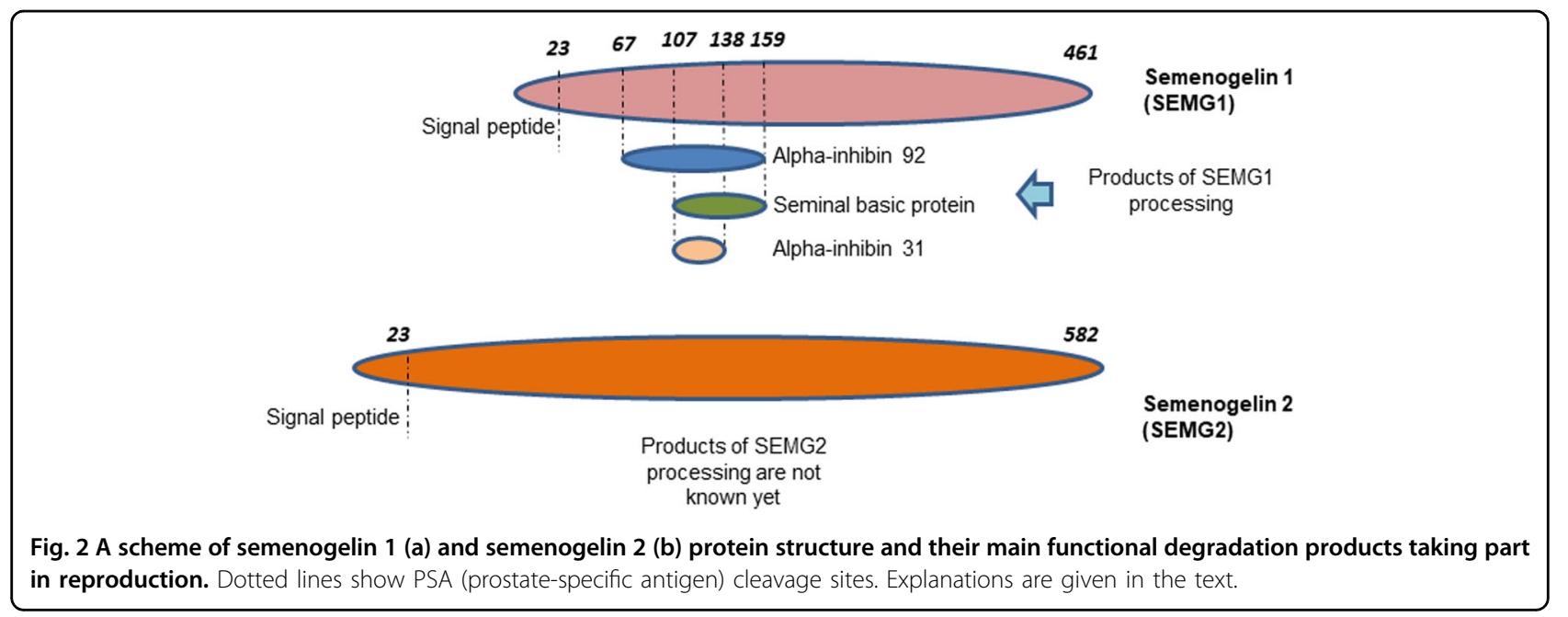

publication that demonstrates a direct mechanism by which one of the CTAs, MAGEC2, affects EMT in breast cancer. This mechanism is realized by MAGEC2 binding to the KAP1 transcriptional repressor, which plays a critical role in promoting the cadherins switch and hence acquiring mesenchymal features by cancer cells ${ }^{23}$. However, a number of other published works listed in Table 1 strongly suggest that the CTAs affect EMT-related processes - migration and invasion when they are accompanied by loss of epithelial and the acquisition of mesenchymal markers.

It should be noted however, that there are reports about CTAs-mediated inhibition of EMT, migration and invasion capabilities of some cancer models ${ }^{24,25}$,. This suggests that the impact of certain CTAs on EMT can depend on the particular cellular context.

\section{SEMG1 and SEMG2: structure and known functions in reproduction}

SEMG1 and SEMG2 are the main proteins of human seminal fluid, which together with fibronectin create the gel-like structure of the ejaculated semen and make up $20-40 \%$ of its volume ${ }^{4,26}$. They are synthesized and secreted mainly by the glandular epithelium of the seminal vesicles. There is evidence that transcripts of SEMGs are also detected in epididymis, vas deferens and prostate, but the functional role of these proteins in these organs is not known today.

Gene encoding SEMGs are localized at the long arm of the 20th chromosome and contain 3 exons. The first exon encodes a signal peptide, the second-the main part of the protein, the third-3'-untranslated region. While the first and third exons of SEMG1 and 2 have conserved sequences, the second exons differ in structure. SEMGs have $78 \%$ homology in protein level. SEMG1 is synthesized as a precursor of 461 a. a. (Fig. 2) and is further processed up to 439 a.a. by cleavage of hydrophobic amino-terminal signal peptide. Thus, the secreted SEMG1 has a molecular weight of $52 \mathrm{kDa}$. In turn, SEMG2 is also synthesized as a precursor of 582 a. a. in size (Fig. 1) followed by cleavage of the amino-terminal signal peptide $^{27,28}$. Secreted SEMG2 consists of 559 a.a. and can be 71 or $76 \mathrm{kDa}$ in size. $71 \mathrm{kDa}$ SEMG2 corresponds to the native protein, while half of all secreted SEMG2 has Nglycosylated asparagine at position 272 (Fig. 2) which corresponds to a molecular weight of $76 \mathrm{kDa}^{27}$.

SEMGs and products of their proteolysis perform a number of important functions in seminal fluid. In particular, they regulate the motility ${ }^{4,29}$, and capacitation of spermatozoa $^{30}$, and provide sperm with antibacterial protection $^{23}$. Although SEMG1 and 2 are highly homologous, almost all information available in literature on the role of SEMGs in reproductive processes concern only SEMG1. The functional role of SEMG2 remains poorly studied even in the reproductive process.

Semenal fluid is a mixture of the seminal vesicle's secretion, and the secretion of prostate and sperm coming from the epididymis ${ }^{31}$. The main function of SEMG1 is to ensure the coagulation of spermatozoa-the compaction of seminal fluid immediately after ejaculation. Then, over time, it gradually liquefies. The coagulation prevents the loss of sperm in the vagina, and its subsequent liquefaction provides sperm the ability move towards the cervix ${ }^{4}$.

During ejaculation, SEMGs and to a lesser extent fibronectin, form a matrix due to non-covalent interactions and disulfide bridges that impede the movement of sperm cells to the ovum ${ }^{32,33}$. Thus, native SEMG1 inhibits sperm motility. Over the next 5-15 min, the proteolysis of SEMG1 occurs followed by liquefaction of the ejaculate and the release of sperm, which then rush to the ovum. Proteolysis of SEMGs is carried out mainly by serine protease, a prostate-specific antigen (PSA), which is part of the secretion of the prostate gland and has chymotrypsin-like proteolytic activity ${ }^{29}$. 


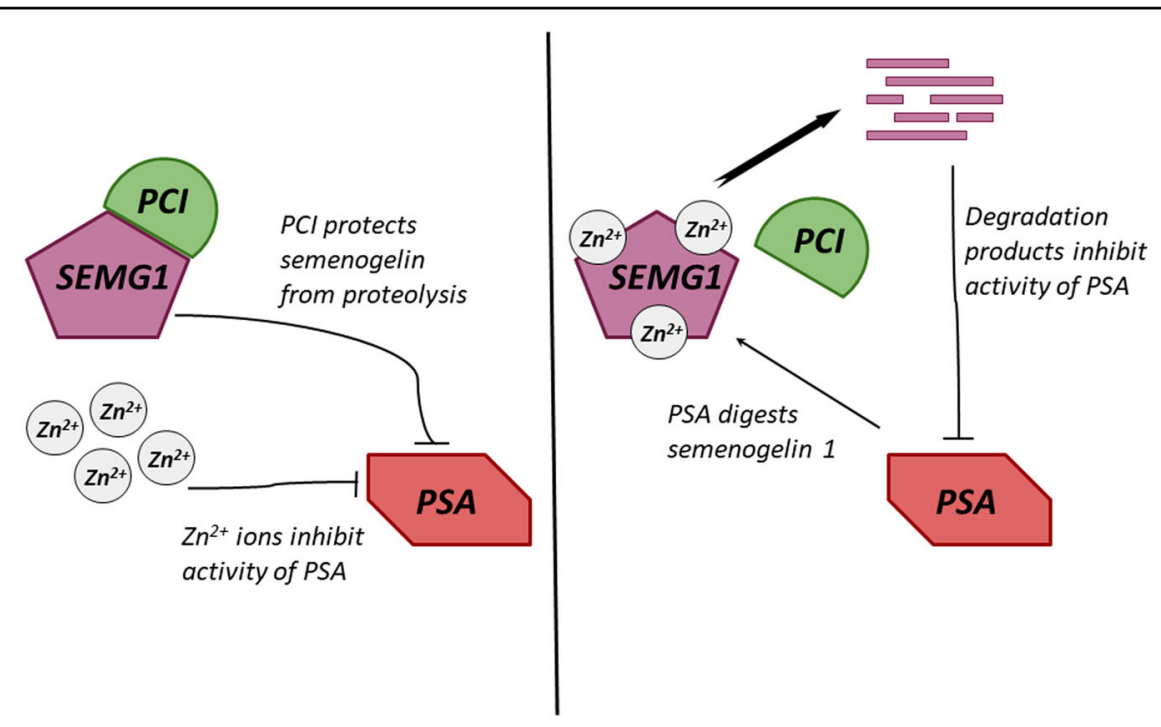

Fig. 3 Regulation of the semenogelin 1 cleavage in seminal vesicles (a) and after ejaculation (b). SEMG1—semenogelin 1, PSA—prostatespecific antigen, PAP_prostatic acid phosphatase, $\mathrm{PCl}$ - protein $\mathrm{C}$ inhibitor. Explanations are given in the text.

To date, three functional peptides have been identified (Fig. 2) obtained as a result of the proteolytic degradation of SEMG1: alpha-inhibin-92, alpha-inhibin- $31^{34}$ and the base protein of seminal fluid ${ }^{35}$. Using a mouse model, it was shown that alpha-inhibin-92 and alpha-inhibin-31 inhibit the secretion of pituitary follicle-stimulating hormone $^{34}$. The data available in literature indicates that SEMG2 is also a substrate for PSA and, accordingly, undergoes fragmentation ${ }^{27,36}$. However, the corresponding peptides resulting from the PSA-dependent cleavage of SEMG2 are not currently described.

The degradation of SEMG1 is regulated by the PCI protein (protein $\mathrm{C}$ inhibitor) (Fig. 3), which, in addition to SEMG1, is also associated with PSA ${ }^{37}$. SEMG1 has the same affinity for both PCI and PSA, and the formation of the corresponding protein complexes depends on the $\mathrm{pH}$, ionic strength, the presence of heparin-like proteins, negatively charged dextran sulfate and divalent cations (in particular zinc). The PCI-SEMG1 complex occurs in the seminal vesicles before ejaculation (Fig. 3), which prevents the proteolytic degradation of SEMG1. During ejaculation, as seminal vesicle secretions move along the ducts, PCI loses the ability to interact with SEMG1 and binds PSA, which, in turn, acquires proteolytitivity and cleaves the SEMG1 into functional oligopeptides (Fig. 3). The PCI-SEMG1 complex is formed at low concentrations of zinc ions in seminal vesicles $(0.86 \mathrm{mM})$, and then dissociates in seminal fluid due to the increase in the concentration of zinc ions $(6.2 \mathrm{mM})^{4}$.

The proteolytic activity of PSA in the seminal fluid is inhibited by zinc ions, while SEMG1is able to chelate these ions (Fig. 3). After ejaculation and SEMG1-mediated zinc binding, PSA activates and cleaves SEMG1 into functional oligopeptides ${ }^{4}$. It was also shown that PCI can form complexes with PSA, thereby most likely, decreasing its proteolytic activity. However, the proportion of such complexes in the seminal fluid is small.

Another important factor of SEMGs-mediated regulation of sperm motility is a serine protease inhibitor Eppin. It is synthesized and excreted by Sertoli cells, as well as epithelial cells of epididymis ${ }^{38}$. Eppin interacts with a protein complex located on the surface of sperm. This complex consists of lactotransferrnin, clusterin, SEMG1 and, accordingly, Eppin ${ }^{39}$. It has been shown that Eppin acts as receptor for SEMG1 and their interaction is extremely important for SG-mediated inhibition of sperm motility. In this case, Eppin regulates the degradation of SEMG1 due to the negative effect on PSA activity. In addition, it was shown that the interaction of SEMG1 with Eppin on the surface of spermatozoa leads to a decrease in intracellular $\mathrm{pH}$ and the absence of oscillations of intracellular calcium, which ultimately hinders sperm motility ${ }^{38}$.

Thus, negative regulation of sperm motility is carried out by both the participation of SEMG1 in the formation of a matrix that impedes their motility, as well as by means of signal cascades unknown so far, initiated after interaction with Eppin and leading to a change in intracellular $\mathrm{pH}$ and calcium waves. Apparently, another function of SEMG1 is the suppression of premature capacitation $^{27}$. Capacitation is the process of sperm maturation in the female genital tract when sperm acquires the ability to penetrate the ovum. It was shown that SEMG1 leads to inhibition of the fertilizing ability of sperm due to the suppression of reactive oxygen species, the generation of which is extremely important for 
capacitation. After getting into the female's genital tract, SEMG1 processing occurs and capacitation becomes possible ${ }^{30}$.

In addition, in vitro experiments have shown that purified SEMG1 and 2 contribute to the activation of testicular hyaluronidase in bovines and humans ${ }^{40}$. Hyaluronidase is secreted by the sperm acrosome in order to hydrolyze hyaluronan, which constitutes a significant part of the intercellular matrix. Thus, activation of hyaluronidase promotes gamete fusion. However, the role of SG in this process in vivo remains unstudied.

Another function of SEMG1 is the antibacterial protection of sperm. SEMG1 itself has antibacterial activity ${ }^{23}$, however products of its processing mediated by PSA ${ }^{41}$ are characterized by significantly more pronounced antimicrobial activity against various bacterial strains.

Thus, SEMGs participate in reproductive function of the body by regulating the motility and maturation of sperm and its protection from bacteria.

\section{Expression of SEMGs in normal tissues and in tumors}

As mentioned above, SEMGs are expressed in germinal tissues. However, a number of research groups have shown their presence at both mRNA and protein levels in several normal tissues ${ }^{5,42}$. In particular, transcripts of SEMGs genes were found in seminal vesicles, seminal ducts, prostate, appendages, trachea, salivary and mammary glands, as well as skin ${ }^{5}$. In this case, the organs of the gastric tract predominantly expressed SEMG1, while in kidneys and testicle the transcript encoding SEMG2 was mainly detected. In addition, mRNAs of SEMGs were detected in skeletal muscle, retina and in the tissues of the central nervous system ${ }^{5}$. Apparently, SEMGs expression is not limited to epithelial tissues and all three germ layers are able to express them.

By using immunohistochemistry, it was shown that prostate tumors more often express both SEMGs than healthy surrounding tissues ${ }^{41}$. In this case, an inverse correlation between SEMG2 expression and the tumor score on the Gleason scale is noted, which indicates the association of SEMG2 with more differentiated tumors. At the same time, in accordance with the Kaplan-Meyer survival curves, the combination of high levels of SEMG1 expression and low levels of SEMG2 is associated with a low survival of patients with prostate tumors ${ }^{43}$.

Predominantly nuclear localization of SEMG1 has been shown in prostate carcinomas in comparison with surrounding healthy tissues ${ }^{44}$. Other researchers have shown that SEMG1 promotes the growth of androgen-sensitive prostate cancer cells, being a zinc-dependent co-activator of the androgen receptor ${ }^{45}$. In addition, SEMG1 protects prostate tumor cells from zinc-mediated cytotoxicity. Thus, literature data indicates the potential difference in the functions of SEMG1 and SEMG2 in prostate cancer.
However, there is evidence of a positive association between SEMG1 level and a patient's survival in case of renal carcinoma. In this case, SEMG1 expression was significantly more often detected in malignant tumors when compared with benign and corresponding normal tissues ${ }^{46}$. In addition, an inverse association was observed between the expression of SEMG1 and the stage of tumor development. According to the Kaplan-Meier survival curves, a low level of SEMG1 was associated with poor patient outcomes ${ }^{46}$. Thus, these data indicate the oncosuppressive properties of SEMG1 in the case of renal carcinomas.

It has been shown that SEMG1 is expressed by tumor cells in chronic myeloid leukemia (CML), chronic lymphoblastic leukemia (CLL) and myeloma ${ }^{47}$. Moreover, the expression of SEMG1 in myeloma cells was increased in the presence of IL- 4 and IL- $6{ }^{48}$. SEMG1 and SEMG2 were detected in a number of cell lines of human lung cancer and melanoma ${ }^{49}$. Immunohistochemistry has shown the expression of SEMGs in 12 of 13 samples of squamous cell lung carcinoma and adenocarcinoma. In addition, SEMGs can also be detected in the blood serum of patients with non-small cell lung cancer ${ }^{50}$ which indicates the possibility of their potential use as biomarkers for the diagnosis of cancer.

\section{Potential biological functions of SEMGs in malignant cells}

As mentioned above, the functions of SEMGs (mainly SEMG1) are known only in reproduction, and information on the functions of SEMGs in malignant neoplasms is particularly absent. An exception is the only study in which SEMG1 was shown as co-activator of the androgen receptor in prostate cancer which protects cancer cells from zinc-mediated cytotoxicity ${ }^{46}$.

However, the association between levels of SEMGs' expression and patient survival can differ depending on the particular tumor type and can be contradictory even in tumors of the same origin. As mentioned above, the expression of SEMG1 in prostate cancer can be associated with both positive and negative patient survival ${ }^{43-46}$. Moreover, SEMG1 and SEMG2 can be contrary associated with the patient's outcome in prostate cancer ${ }^{43}$. It is possible to suggest that SEMG1 and SEMG2 may have different functions even in the same cells. This suggestion is supported by our data about the different spectrum of SEMGs' interactants in MCF7 breast carcinoma cell model identified by LC-MS/MS (Shuvalov et al., in press). In the same research, we have also shown that SEMGs can up-regulate energy metabolism of cancer cell models by increasing glycolysis and respiration.

Zhang with colleagues ${ }^{46}$ have shown a positive association between the level of SEMG1 and a patient's survival in the case of renal carcinoma. We have also demonstrated that SEMGs possess different anti- 
proliferative properties of lung adenocarcinoma H1299 cells $^{51}$. Apparently, SEMGs can possess both oncogenic and oncosupressive features depending on the particular cellular background.

Since SEMGs belong to CTAs and are normally expressed primarily in germ cells, the legitimate question is whether SEMGs perform any functions in tumor cells or they can be just cancer bystanders, i.e. genes whose expression is restored in cancer cells as part of the larger embryonic genes signature re-activation program. It is well known that tumors, especially the lowdifferentiated ones, often express a gene pattern similar to embryonic stem cells ${ }^{52-54}$. Germ cell precursors, like trophoblast cells have much in common with tumor cells $^{2}$. For example, in the process of colonization of gonad primordia, germ cells are very mobile and have the ability to invade tissues ${ }^{55}$. During spermatogenesis, spermatogonia maintain proliferative activity throughout their life. In turn, trophoblast cells are able to invade the endometrium and actively proliferate, forming part of the placenta. All these are very similar to properties of tumor cells. Thus, neoplastic cells have much in common with germ cells ${ }^{53,54}$ and from this point of view it is possible to explain why they express a similar gene pattern.

Taking into account our data about SEMGs' features in several cancer cell models, literature data about biological activity of SEMG1 in prostate cancer and the known biological role of SEMGs in reproduction, we hypothesize that SEMGs expression can have an impact on malignant cells. Also, it is interesting to note that the biological role for a number of CTAs in tumor cells has been described. In particular, CTAs can promote proliferation (SSX2, CAGE), genomic instability (FMR1NB, NXF2, SSX2), evasion from apoptosis (MAGE-A, MAGE-B, MAGE-C, CAGE, PAGE4), induction of angiogenesis (CAGE), invasion and metastasis (MAGE-C2) ${ }^{2}$.

For example, there are two well-known oncofetal proteins, Carcinoembryonic antigen (CEA) and alphafetoprotein (AFP), which originate within tumor cells and enter the bloodstream either by secretion from the tumor or as a breakdown product of tumor cells. Normally they are expressed during embryogenesis and then become epigenetically silenced. Importantly, their expression may be re-activated in certain cancers making them potentially useful tumor markers. Furthermore, there are evidences demonstrating the active roles in carcinogenesis for both of them ${ }^{56}$. AFP was shown to function as a growth regulator by binding to key proteins involved in signaling pathways. In particular, AFP has been demonstrated to block the RA-RAR signaling to disrupt the forward transmission of apoptotic signaling $^{57,58}$ AFP also interacts with PTEN to activate the PI3K/AKT pathway, leading to aberrant growth and migration of HCC cells ${ }^{49,59-61}$. Furthermore, AFP inhibits autophagy to promote malignant behavior in hepatocellular carcinoma cells by activating PI3K/AKT/mTOR signaling ${ }^{62}$. Tumor-derived AFP impairs the differentiation and T-cell stimulatory activity of human dendritic cells ${ }^{63}$. Taking all these facts together, it would be fair to say that at least some of the re-activated CTAs play active roles in tumorigenesis.

As discussed above, SEMGs regulate the motility of sperm (create a gel-like matrix, activate hyaluronase), regulate its maturation (are scavengers of reactive oxygen species during capacitation) and participate in zinc homeostasis. These functions can also occur in neoplastic cells. For instance, the motility of a tumor cell is the basis of invasion and metastasis ${ }^{64}$. At the same time, rapidly proliferating cancer cells are under constitutive oxidative stress $^{65}$. Moreover, zinc is capable of exerting a strong toxic effect on tumor cells of various origins in comparison with normal cells; in addition, the concentration of zinc ions in tumor tissues is often reduced ${ }^{66}$. Thus, the already known functions of SEMGs in reproduction can be used by tumor cells to maintain their vital functions. However, each of these potential molecular mechanisms requires a separate study.

In addition, SEMGs can possess some other molecular functions in cancer cells unknown yet. According to the BioGrid database (https://thebiogrid.org/), SEMGs interact with more than twenty different proteins, among which there are regulators of the cell cycle, apoptosis, etc. The functional role of their interaction with SEMGs remains to be investigated.

\section{Conclusion and perspectives}

SEMGs are CTAs which are expressed in malignancies of various origins. However, their biological functions were studied exclusively in the context of the reproductive function of the body. So, this is important to ask about the functions of SEMGs in malignant cells.

Do SEMGs undergo processing and do their functions in cancer cells depend on proteases? What are molecular and phenotypic changes in tumor cells during SEMGs expression or silencing? What are the molecular mechanisms mediating this effect? Answers to these questions will help clarify the functional role of SEMGs in oncogenesis.

Also, questions about the immunogenicity of SEMGs and their possible role in the antitumor immune response are extremely important. It has been shown that in a number of cases of CML and CLL expressing SEMG1, antibodies specific to SEMG1 were detected in patients ${ }^{4}$. It means that in patients with tumor expressing SEMGs, the immune response against these proteins may occur, which contributes to the destruction of tumor cells by $\mathrm{T}$ lymphocytes. 
However, in another work ${ }^{67}$ it was shown that SEMG1 can inhibit the proliferation of peripheral blood lymphocytes and the production of immunoglobulins. Thus, SEMGs can potentially be involved in the antitumor immune response. So, the assessment of SEMGs expression in tumors and their occurrence in the serum of cancer patients, as well as the study of their functions can be of clinical significance in the context of identifying new tumor markers and the development of new approaches to antitumor immunotherapy.

\section{Acknowledgements}

This work was supported by the Russian Science Foundation grant no. 19-4502011 (O.S., C.D., O.F. and N.B.). O.S. and A.K. appreciate the support of the grant from the Russian Government Program for the Recruitment of the leading scientists into the Russian Institutions of Higher Education 14. W03.31.0029. N.B. appreciates the support of RFBR, project \#18-29-09144.

\section{Author details}

${ }^{1}$ Institute of cytology RAS, St-Petersburg, Russia. ${ }^{2}$ Almazov Federal North-West Medical Research Centre, St-Petersburg, Russia. ${ }^{3}$ MIPT, Dolgoprudny, Moscow region, Moscow, Russia

\section{Conflict of interest}

The authors declare no conflict of interest.

\section{Publisher's note}

Springer Nature remains neutral with regard to jurisdictional claims in published maps and institutional affiliations.

Received: 12 December 2019 Revised: 22 November 2020 Accepted: 17 January 2021

Published online: 08 May 2021

\section{References}

1. da Silva, V. L. et al. Genome-wide identification of cancer/testis genes and their association with prognosis in a pan-cancer analysis. Oncotarget 8, 92966 (2017).

2. Gjerstorff, M. F., Andersen, M. H. \& Ditzel, H. J. Oncogenic cancer/testis antigens: prime candidates for immunotherapy. Oncotarget 6, 15772 (2015).

3. Krishnadas, D. K., Bai, F. \& Lucas, K. G. Cancer testis antigen and immunotherapy. ImmunoTargets Ther. 2, 11 (2013).

4. De Lamirande, E. in Seminars in thrombosis and hemostasis. 060-068 (Copyright@ 2007 by Thieme Publishers, Inc., 333 Seventh Avenue, New York, USA).

5. Lundwall, A., Bjartell, A., Olsson, A. Y. \& Malm, J. Semenogelin I and II, the predominant human seminal plasma proteins, are also expressed in nongenital tissues. Mol. Hum. Reprod. 8, 805-810 (2002).

6. Vigneron, N. Human tumor antigens and cancer immunotherapy. BioMed Res. Int. 2015, 1-17 (2015).

7. Le Naour, F., Brichory, F., Beretta, L. \& Hanash, S. M. Identification of tumorassociated antigens using proteomics. Technol. Cancer Res. Treat. 1, 257-262 (2002).

8. Simpson, A. J., Caballero, O. L., Jungbluth, A., Chen, Y.-T. \& Old, L. J. Cancer/ testis antigens, gametogenesis and cancer. Nat. Rev. Cancer 5, 615 (2005).

9. Caballero, O. L. \& Chen, Y. T. Cancer/testis (CT) antigens: potential targets for immunotherapy. Cancer Sci. 100, 2014-2021 (2009).

10. Andrade, V. C. et al. Prognostic impact of cancer/testis antigen expression in advanced stage multiple myeloma patients. Cancer Immun. Arch. 8, 2 (2008).

11. Costa, F. F., Le Blanc, K. \& Brodin, B. Concise review: cancer/testis antigens, stem cells, and cancer. Stem Cells 25, 707-711 (2007).

12. Fratta, E. et al. The biology of cancer testis antigens: putative function, regulation and therapeutic potential. Mol. Oncol. 5, 164-182 (2011).
13. Almeida, L. G. et al. CTdatabase: a knowledge-base of high-throughput and curated data on cancer-testis antigens. Nucleic Acids Res. 37, D816-D819 (2008).

14. Stevenson, B. J. et al. Rapid evolution of cancer/testis genes on the X chromosome. BMC Genomics 8, 129 (2007).

15. Gibbs, Z. A. \& Whitehurst, A. W. Emerging contributions of cancer/testis antigens to neoplastic behaviors. Trends Cancer 4, 701-712 (2018).

16. Maxfield, K. E. et al. Comprehensive functional characterization of cancer-testis antigens defines obligate participation in multiple hallmarks of cancer. Nat. Commun. 6, 1-15 (2015).

17. Roche, J. (Multidisciplinary Digital Publishing Institute, 2018).

18. Cho, E. S., Kang, H. E., Kim, N. H. \& Yook, J. I. Therapeutic implications of cancer epithelial-mesenchymal transition (EMT). Arch. Pharmacal. Res. 42, 14-24 (2019).

19. Ribatti, D., Tamma, R. \& Annese, T. Epithelial-mesenchymal transition in cancer: a historical overview. Transl. Oncol. 13, 100773 (2020).

20. Smith, B. N. \& Bhowmick, N. A. Role of EMT in metastasis and therapy resistance. J. Clin. Med. 5, 17 (2016).

21. Shibue, T. \& Weinberg, R. A. EMT, CSCS, and drug resistance: the mechanistic link and clinical implications. Nat. Rev. Clin. Oncol. 14, 611 (2017).

22. Yang, P., Huo, Z., Liao, H. \& Zhou, Q. Cancer/testis antigens trigger epithelialmesenchymal transition and genesis of cancer stem-like cells. Curr. Pharm. Des. 21, 1292-1300 (2015)

23. Bourgeon, F. et al. Involvement of semenogelin-derived peptides in the antibacterial activity of human seminal plasma. Biol. Reprod. 70, 768-774 (2004).

24. Wang, $X$. et al. Silencing HIWI suppresses the growth, invasion and migration of glioma cells. Int. J. Oncol. 45, 2385-2392 (2014).

25. Hu, Y. et al. RGS22, a novel cancer/testis antigen, inhibits epithelial cell invasion and metastasis. Clin. Exp. Metastasis 28, 541-549 (2011).

26. Lilja, H., Abrahamsson, P.-A. \& Lundwall, A. Semenogelin, the predominant protein in human semen. Primary structure and identification of closely related proteins in the male accessory sex glands and on the spermatozoa. J. Biol. Chem. 264, 1894-1900 (1989).

27. Kise, H., Nishioka, J., Kawamura, J. \& Suzuki, K. Characterization of semenogelin $\|$ and its molecular interaction with prostate-specific antigen and protein C inhibitor. Eur. J. Biochem. 238, 88-96 (1996).

28. Lilja, H. \& Lundwall, A. Molecular cloning of epididymal and seminal vesicular transcripts encoding a semenogelin-related protein. Proc. Natl Acad. Sci. USA 89, 4559-4563 (1992).

29. Robert, M., Gibbs, B. F., Jacobson, E. \& Gagnon, C. Characterization of prostatespecific antigen proteolytic activity on its major physiological substrate, the sperm motility inhibitor precursor/semenogelin I. Biochemistry 36, 3811-3819 (1997).

30. de Lamirande, E., Yoshida, K., Yoshiike, M., Iwamoto, T. \& Gagnon, C. Semenogelin, the main protein of semen coagulum, inhibits human sperm capacitation by interfering with the superoxide anion generated during this process. J. Androl. 22, 672-679 (2001).

31. Rodríguez-Martínez, H., Kvist, U., Ernerudh, J., Sanz, L. \& Calvete, J. J. Seminal plasma proteins: what role do they play? Am. J. Reprod. Immunol. 66, 11-22 (2011).

32. Lwaleed, B. A., Greenfield, R., Stewart, A., Birch, B. \& Cooper, A. J. Seminal clotting and fibrinolytic balance: a possible physiological role in the male reproductive system. Thrombosis Haemost. 92, 752-766 (2004).

33. Robert, M. \& Gagnon, C. Purification and characterization of the active precursor of a human sperm motility inhibitor secreted by the seminal vesicles: identity with semenogelin. Biol. Reprod. 55, 813-821 (1996).

34. Li, C. H., Hammonds, R. G., Ramasharma, K. \& Chung, D. Human seminal alpha inhibins: isolation, characterization, and structure. Proc. Natl Acad. Sci. USA 82, 4041-4044 (1985).

35. Lilja, H. \& Jeppsson, J.-O. Amino acid sequence of the predominant basic protein in human seminal plasma. FEBS Lett. 182, 181-184 (1985).

36. Lilja, H. \& Laurell, C.-B. Liquefaction of coagulated human semen. Scand. J. Clin. Lab. Investig. 44, 447-452 (1984).

37. Suzuki, K., Kise, H., Nishioka, J. \& Hayashi, T. in Seminars in thrombosis and hemostasis. 046-052 (Copyright@ 2007 by Thieme Publishers, Inc., 333 Seventh Avenue, New York, NY).

38. O'Rand, M., Widgren, E., Wang, Z. \& Richardson, R. Eppin: an epididymal protease inhibitor and a target for male contraception. Soc. Reprod. Fertil. suppl. 63, 445-453 (2007). 
39. Mitra, A., Richardson, R. T. \& O'Rand, M. G. Analysis of recombinant human semenogelin as an inhibitor of human sperm motility. Biol. Reprod. $\mathbf{8 2}$ 489-496 (2010).

40. Mandal, A. \& Bhattacharyya, A. K. Andrology: sperm hyaluronidase activation by purified predominant and major basic human seminal coagulum proteins. Hum. Reprod. 10, 1745-1750 (1995).

41. Edström, A. M. et al. The major bactericidal activity of human seminal plasma is zinc-dependent and derived from fragmentation of the semenogelins. J. Immunol. 181, 3413-3421 (2008).

42. Jonsson, M., Lundwall, A. \& Malm, J. The semenogelins: proteins with functions beyond reproduction? Cell. Mol. Life Sci. CMLS 63, 2886-2888 (2006).

43. Canacci, A. M. et al. Expression of semenogelins I and II and its prognostic significance in human prostate cancer. Prostate 71, 1108-1114 (2011).

44. Izumi, K. et al. Seminal plasma proteins in prostatic carcinoma: increased nuclear semenogelin I expression is a predictor of biochemical recurrence after radical prostatectomy. Hum. Pathol. 43, 1991-2000 (2012).

45. Ishiguro, $\mathrm{H}$. et al. Semenogelin I promotes prostate cancer cell growth via functioning as an androgen receptor coactivator and protecting against zinc cytotoxicity. Am. J. Cancer Res. 5, 738 (2015).

46. Zhang, S. et al. Seminal plasma protein in renal cell carcinoma: expression of semenogelin I is a predictor for cancer progression and prognosis. Tumor Biol. 35, 9095-9100 (2014).

47. Zhang, Y., Wang, Z., Liu, H., Giles, F. J. \& Lim, S. H. Pattern of gene expression and immune responses to Semenogelin 1 in chronic hematologic malignancies. J. Immunother. 26, 461-467 (2003).

48. Zhang, Y., Wang, Z., Zhang, J., Farmer, B. \& Lim, S. H. Semenogelin I expression in myeloma cells can be upregulated pharmacologically. Leuk. Res. 32, 1889-1894 (2008).

49. Zhu, M. et al. HBx drives alpha fetoprotein expression to promote initiation of liver cancer stem cells through activating PI3K/AKT signal pathway. Int. J. Cancer 140, 1346-1355 (2017).

50. Rodrigues, R. G. et al. Semenogelins are ectopically expressed in small cell lung carcinoma. Clin. Cancer Res. 7, 854-860 (2001).

51. Shuvalov, O. et al. Cancer-testis antigens, semenogelins 1 and 2, exhibit different anti-proliferative effects on human lung adenocarcinoma cells. Cell Death Discov. 6, 1-8 (2020).

52. Ben-Porath, I. et al. An embryonic stem cell-like gene expression signature in poorly differentiated aggressive human tumors. Nat. Genet. 40, 499 (2008).

53. Cofre, J. \& Abdelhay, E. Cancer is to embryology as mutation is to genetics: hypothesis of the cancer as embryological phenomenon. Sci. World J. 2017 $1-17$ (2017)
54. Old, L. J. (AACR, 2007)

55. Mamsen, L. S., Brøchner, C. B., Byskov, A. G. \& Møllgard, K. The migration and loss of human primordial germ stem cells from the hind gut epithelium towards the gonadal ridge. Int. J. Dev. Biol. 56, 771-778 (2013).

56. Rizeq, B., Zakaria, Z. \& Ouhtit, A. Towards understanding the mechanisms of actions of carcinoembryonic antigen-related cell adhesion molecule 6 in cancer progression. Cancer Sci. 109, 33-42 (2018).

57. Wang, S. et al. Alpha-fetoprotein acts as a novel signal molecule and mediates transcription of Fn14 in human hepatocellular carcinoma. J. Hepatol. 57 322-329 (2012)

58. Zhang, $C$. et al. Alpha fetoprotein mediates $H B \times$ induced carcinogenesis in the hepatocyte cytoplasm. Int. J. Cancer 137, 1818-1829 (2015).

59. Li, M. et al. Alpha-fetoprotein: a new member of intracellular signal molecules in regulation of the PI3KJAKT signaling in human hepatoma cell lines. Int. J. Cancer 128, 524-532 (2011).

60. Zhu, M. et al. Alpha-fetoprotein activates AKT/mTOR signaling to promote CXCR4 expression and migration of hepatoma cells. Oncoscience 2, 59 (2015).

61. Zhu, M. et al. Hepatitis B virus $X$ protein induces expression of alphafetoprotein and activates PI3K/mTOR signaling pathway in liver cells. Oncotarget 6, 12196 (2015).

62. Wang, S. et al. Alpha-fetoprotein inhibits autophagy to promote malignant behaviour in hepatocellular carcinoma cells by activating PI3K/AKT/mTOR signalling. Cell Death Dis. 9, 1-13 (2018).

63. Pardee, A. D., Shi, J. \& Butterfield, L. H. Tumor-derived a-fetoprotein impairs the differentiation and $\mathrm{T}$ cell stimulatory activity of human dendritic cells. J. Immunol. 193, 5723-5732 (2014).

64. Riggi, N., Aguet, M. \& Stamenkovic, I. Cancer metastasis: a reappraisal of its underlying mechanisms and their relevance to treatment. Annu. Rev. Pathol. Mechanisms Dis. 13, 117-140 (2018).

65. Sosa, V. et al. Oxidative stress and cancer: an overview. Ageing Res. Rev. 12 376-390 (2013).

66. Costello, L. C. \& Franklin, R. B. Decreased zinc in the development and progression of malignancy: an important common relationship and potential for prevention and treatment of carcinomas. Expert Opin. Ther. Targets 21, 51-66 (2017).

67. Matsushita, T. et al. Evidence for immunosuppressive effects of human semenogelin, a major protein of seminal plasma. St. Marianna Med. J. 31, 73-82 (2003). 